\title{
UM ESTUDO SOBRE A SAÚDE DA POPULAÇÃO NEGRA NO BRASIL: NA
} BUSCA POR EQUIDADE

\section{Luiza Constante Oliveira'; Alini Basso de Souza ${ }^{2}$; Giovana Durigon Alves; Liliane Tomazi Vestena ${ }^{4}$; Luiza Nicole Lazzaretti ${ }^{5}$; Suélen Cossettin Battisti ${ }^{6}$; Fernanda Pires Jaeger ${ }^{7}$}

\section{RESUMO}

O presente artigo objetiva-se analisar as políticas de saúde voltadas para a população negra no Brasil. Além de compreender seu funcionamento e os possíveis desafios do acesso dessa população ao Sistema de Saúde, investigando os impactos do racismo na saúde mental. Esse trabalho trata-se de uma pesquisa bibliográfica de cunho qualitativo. A coleta de dados realizou-se nos meses de março e abril de 2021 nas bases de dados Scielo (Scientific Electronic Library), Pepsic (Periódicos Eletrônicos em Psicologia), revistas, livros, repositórios de Universidades, manuais do Ministério da Saúde e no Instituto Brasileiro de Geografia e Estatística (IBGE). Pode-se notar que o Brasil já evoluiu bastante frente às questões raciais, porém algumas delas ainda estão estruturadas na sociedade, assim, é perceptível os desafios enfrentados pela população negra no acesso à saúde. Conclui-se que é necessária a implementação de políticas necessárias e resolutivas, para que o direito à saúde da população negra seja garantido.

Palavras-chave: Negros; Políticas de saúde; Racismo; Saúde mental; Sistema Único de Saúde (SUS).

Eixo Temático: Atenção Integral e Promoção à Saúde.

\section{INTRODUÇÃO}

Segundo o Instituto Brasileiro de Geografia e Estatística (IBGE), de acordo com a Pesquisa Nacional de Saúde (PNS) realizada em 2013, a porcentagem de pessoas que se autodeclararam negras (pretas ou pardas) chegava a $53,9 \%$, sendo mais da metade da população do Brasil, o que aponta para um aumento nessa porcentagem nos últimos anos (IBGE, 2015). Além disso, é perceptível que houve

\footnotetext{
${ }^{1}$ Luiza Constante Oliveira - Universidade Franciscana - luiza.constante@ufn.edu.br.

${ }^{2}$ Alini Basso de Souza - Universidade Franciscana - alini.basso@ufn.edu.br.

${ }^{3}$ Giovana Durigon Alves - Universidade Franciscana - giovana.durigon@ufn.edu.br.

${ }^{4}$ Liliane Tomazi Vestena - Universidade Franciscana - liliane.tomazi@ufn.edu.br.

${ }^{5}$ Luiza Nicole Lazzaretti - Universidade Franciscana - luiza.nicole@ufn.edu.br.

6 Suélen Cossetin Battisti - Universidade Franciscana - suelen.cossettin@ufn.edu.br.

7 Fernanda Pires Jaeger - Universidade Franciscana - fpjaeger@ufn.edu.br.
} 
um aumento na renda média per capita desses indivíduos, porém ainda está longe de se igualar ao da população branca. Essas desigualdades aparecem também em outras esferas sociais, como na saúde, pois esses indicadores em conjunto com as questões econômicas apontam que existe uma importante ligação entre as diferenças sociais, como o sistema de saúde se estrutura e a saúde dessas populações (BRASIL, 2017).

Levando em consideração que as iniquidades existentes na saúde atualmente são produtos de processos socioeconômicos e culturais, é necessário a criação de políticas e programas voltados para os diferentes grupos sociais para que se possa cumprir o princípio de equidade assegurado pelo Sistema Único de Saúde (SUS). Pensando nisso, em 2009, o Ministério de Saúde, juntamente com o Pacto pela Saúde e a Política Nacional de Gestão Estratégica e Participativa no SUS (ParticipaSUS), desenvolveu a Política Nacional de Saúde Integral da População Negra (PNSIPN), a qual visa superar os obstáculos que afetam de forma negativa a saúde dessa população (BRASIL, 2017).

Além disso, o Cômite Técnico de Saúde da População Negra foi criado pelo Ministério da Saúde tendo como objetivo promover uma equidade de saúde para os negros. Logo, isso só ocorreu depois de muita luta e mobilização por meio, por exemplo, da III Conferência Internacional contra o Racismo, Homofobia e as Intolerâncias Correlatas. Desse modo, destaca-se ainda, atualmente, após essas diversas políticas de saúde implantadas dentro dos serviços de saúde um racismo institucional que impossibilita que as instituições possam promover os direitos adequadamente e romper com as desigualdades sociais, contribuindo para a exclusão dessa população racialmente subordinada (FAUSTINO, 2017).

Embora existam diversas políticas públicas e ações para melhorar o acesso da população negra aos serviços de saúde, ainda existe uma precariedade no monitoramento e avaliação das mesmas, o que acaba prejudicando a aplicação dessas medidas. Com isso, os fatores que dificultam a busca deste grupo pelos serviços de saúde se fazem presentes no cenário atual, podendo se dar de 
diferentes formas, seja através do desrespeito à diversidade cultural, racial e étnica, a partir da atuação dos profissionais, ou ainda por barreiras estruturais, questões sociais e econômicas (SILVA et al., 2020).

Diante desse contexto e das diversas iniquidades que ainda perpassam o sistema de saúde no Brasil, se faz necessário o desenvolvimento de estudos que abordem esse tema. Por isso o presente trabalho justifica-se pela relevância em abordar a temática sobre a saúde da população negra, bem como a busca de espaços de diálogo e disseminação de conhecimentos relacionados ao tema que possam orientar essa população. Logo, objetiva-se analisar o contexto de políticas de saúde voltadas para a população negra no Brasil, como também compreender o seu funcionamento, buscar conhecer os possíveis desafios do acesso da população negra ao Sistema de Saúde e investigar os impactos que o racismo pode causar na saúde mental dos indivíduos negros.

\section{METODOLOGIA}

A estruturação do presente artigo foi realizada a partir dos moldes de uma pesquisa bibliográfica de cunho qualitativo, tratando-se de um estudo não-sistemático, no qual não foi necessário a utilização dos critérios de restrição por idioma, data e local de publicação, sendo exigido apenas que os dados se encaixam no tema proposto. Além disso, a coleta de dados foi realizada durante os meses de março e abril de 2021, a partir de obras encontradas na base de dados Scielo (Scientific Electronic Library), Pepsic (Periódicos Eletrônicos em Psicologia), revistas, livros, repositórios de Universidades Brasileiras, manuais do Ministério da Saúde e no Instituto Brasileiro de Geografia e Estatística (IBGE). Com isso, os dados coletados foram analisados a partir da perspectiva do método de Análise de Conteúdo conforme Bardin (2016), o qual foi organizado em um período inicial de pré-análise, seguido por uma exploração do material e, por último, pelo tratamento dos resultados. 
A pré-análise visa escolher documentos, formular hipóteses e objetivos, além de elaborar uma interpretação final, organizando assim, os materiais que serão utilizados neste artigo. Durante a exploração do material, aplica-se às decisões tomadas na fase anterior, ou seja, ela é a análise propriamente dita. Por fim, a última fase é intitulada como tratamento dos resultados, visando a interpretação dos materiais obtidos (BARDIN, 2016). A partir da análise realizada elaborou-se as seguintes categorias: O contexto das políticas de saúde voltadas para a população negra no Brasil; Algumas dificuldades enfrentadas pelas pessoas negras no acesso ao sistema de saúde; Os impactos que o racismo pode causar na saúde mental dos individuos negros conforme visto a seguir.

\section{RESULTADOS E DISCUSSÕES}

\section{1 contexto das políticas de saúde voltadas para a população negra no}

\section{Brasil}

Segundo uma pesquisa realizada pela PNS em 2013, no qual investigou-se os aspectos sobre a cobertura de saúde suplementar, considerando a proporção da população que possui acesso ao serviço de planos de saúde, foi apontado que apenas $21,6 \%$ da população negra possuía acesso a esse tipo de serviço. Dessa forma, o baixo acesso à saúde por parte da população negra significa uma exposição maior a riscos e as desigualdades sociais vivenciadas por essa população são expressadas nesses dados.

Além disso, vale destacar que a $8^{\circ}$ Conferência Nacional de Saúde foi de extrema importância, pois ela trouxe a oportunidade de que a ideia de uma política voltada para a população negra se consolidasse em um futuro próximo, já que na sua estruturação haviam particularidades da população negra, que estavam sendo representadas pelo movimento negro (COSTA, 2019). Dessa forma, os membros desse movimento representaram a população, lutando pelas suas necessidades de saúde e qualidade de atendimento, assim como a equidade nos serviços que se concretizaram através de políticas públicas implementadas para essa população. 
Dessa forma, as políticas públicas desenvolvidas têm contribuído de forma significativa para o combate da vulnerabilidade e desigualdade no Brasil. Assim, o Ministério da Saúde elaborou a PNSIPN, sendo um compromisso firmado entre as esferas federal, estadual e municipal. A PNSIPN foi implementada pela portaria $\mathrm{n}^{\circ}$ 992, de 13 de maio de 2009 e possui como marca a identificação do racismo, das desigualdades étnico-raciais e do racismo institucional como causa social das condições de saúde e dispõe como objetivo geral a promoção da integralidade da saúde da população negra, priorizando a redução das desigualdades, da discriminação étnico-raciais e do combate ao racismo nas instituições e serviços do SUS (BRASIL, 2009).

Neste viés, segundo Costa (2019) uma grande parcela das diretrizes que constituem as estratégias de ferramentas para a saúde dessa população não fora concretizada no sistema de saúde, mas por indivíduos negros que juntos organizaram-se a partir de movimentos sociais, para a construção de novos conhecimentos e perpetuação de seus valores. Porém, a igualdade social não será facilmente alcançada, do ponto de vista histórico brasileiro e de sua construção em sociedade. Visto isso, as políticas públicas voltadas para a garantia dos direitos são fundamentais como ferramentas na luta dos movimentos sociais contra as discriminações e preconceitos.

Ademais, vale salientar que o racismo está diretamente ligado à qualidade de atendimento de saúde que as pessoas negras recebem e, consequentemente, ao adoecimento e a morte dessa população. Visto isso, segundo Mesquita (2019) é necessário que as instituições de saúde estejam atentas aos efeitos das discriminações nas relações. Segundo o autor, as ações voltadas para o enfrentamento do racismo, o combate às iniquidades no sistema de saúde e o cuidado com a saúde dessa população também são responsabilidade da atenção básica, que deve realizar ações voltadas para o cuidado desses indivíduos. É importante que cada vez mais existam estratégias de promoção à saúde, que visem 
estimular a aproximação da população com elementos da sua cultura, para que se exerça um combate ao racismo.

\subsection{Algumas dificuldades enfrentadas pelas pessoas negras no acesso ao sistema de saúde}

Em um estudo realizado por Kalckmann et al. (2007) foi coletado relatos de usuários negros que já sofreram discriminação em algum serviço de saúde por parte de diversos profissionais das unidades. Os relatos evidenciaram nas falas dos profissionais a discriminação e os preconceitos atrelados a formas diversificadas de afastar o usuário dos serviços de saúde, como "negros são mais resistentes a dor e não adocem" ou em situações em que os profissionais de saúde negligenciaram e menosprezaram a dor dos individuos negros, com as seguintes expressões: "Rapaz! Um negão desse tamanho sentindo dor?" "O que é isso? Não dói tanto", tendo a tendência de minimizar as dores e queixas desses pacientes com palavras discriminatórias dirigido a eles.

Segundo o mesmo autor, também foi constatado preconceito dirigido aos profissionais negros, apontando para o rascismo institucional na hora da contratação e por parte dos pacientes. Muitos dos entrevistandos relataram não terem sido contratados por serem negros e que os cargos de maior poder, como a direção, são designados às pessoas brancas, assim como pacientes se negaram a serem atendidos por indivíduos negros. De acordo com Guimarães (2000) a razão do racismo institucional, através de insultos pode ser variável, mas está ligado a uma questão de poder, com a tentativa de legitimar uma hierarquia social baseada na ideia de raça, na qual uma prevalece sobre a outra.

As iniquidades na saúde, agravam o adoecimento da população negra no contexto de pandemia de COVID-19, gerando um aumento na taxa de mortalidade dessa população. Além disso, o racismo estrutural praticado em instituições de saúde, têm contribuindo para esse agravamento, pois interfere diretamente na aplicabilidade da atenção integral e equidade. Esses fatos salientam que existe um 
privação dessa população aos serviços de saúde e a desumanização no atendimento, além de situação de baixa renda de muitos desses indivíduos, reflete na dificuldade de enfrentamento da pandemia por esse grupo populacional. Ademais, é perceptível que os indivíduos negros sofrem maior impacto com a pandemia, portanto, compreende-se que a COVID-19 afeta de maneira desigual os grupos sociais (SANTOS et al, 2021).

Cabe salientar que os profissionais da saúde devem trabalhar e lutar contra qualquer tipo de discriminação, evitando a disseminação desse tipo de violência, considerando sempre que racismo é crime. Dessa forma, as desigualdades na saúde serão amenizadas e com uma luta constante podem até ser extintas, trazendo uma melhora na atenção à saúde das pessoas negras. A equidade na saúde desses indivíduos será atingida, quando os profissionais colocarem-na em prática no cotidiano, tendo em vista que o contrário é considerado crime (COSTA, 2019). De acordo com o autor, o racismo é uma das doenças que atingem a maior parte da população brasileira, portanto, é imprescindível para a construção de uma melhor qualidade de saúde e de vida, que o racismo seja extinguido da sociedade.

\subsection{Os impactos que o racismo pode causar na saúde mental dos individuos negros}

De acordo com Gonçalves et al. (2020), diferente do que muitos indivíduos acreditam, o racismo não foi extinto, mas sim mascarado, ele pode não ser tão visível aos olhos, mas está encoberto dentro da sociedade. As pessoas da raça negra estão sujeitas a muitos constrangimentos como humilhações, piadas e brincadeiras racistas, essas situações influenciam diretamente na autoestima. Além disso, a ocorrência de violência, estereótipos, discriminação e falta de representatividade são causadores de transtornos mentais e traumas nessa população.

Conforme os dados mostrados na cartilha "Óbitos por suicídio entre adolescentes e jovens negros 2012 a 2016" divulgadas em 2018 pelo Ministério da 
Saúde, a quantidade de jovens e adolescentes que acabaram tirando a própria vida é superior na população negra. O documento indica também que a proporção de suicídios entre negros aumentou de 2012 a 2016 em relação às outras etnias, passando de $53,3 \%$ para $55,4 \%$. Além disso, a cartilha atenta para um risco maior de vulnerabilidade psicológica, pois esse grupo enfrenta diariamente problemas como racismo institucional, preconceito e discriminação (BRASIL, 2018).

Portanto, é perceptível que o racismo gera grande impacto na saúde mental. Em uma pesquisa realizada por esses autores, foi relatado que os entrevistados da raça negra, quando estão em processo de terapia, se sentem mais confortáveis para abordar assuntos raciais com profissionais negros, comparado aos profissionais brancos, pois tem a crença de que os terapeutas negros já passaram por situações de racismo, podendo compreende-los melhor, isso evidencia a importância de o paciente encontrar um profissional com o qual possa se identificar (GONÇALVES et al 2020).

Segundo Lages et al. (2014), assim como as doenças físicas acometem o corpo do ser humano, a subjetividade do indivíduo também pode ser afetada devido aos preconceitos e ao demérito das suas identidades culturais, havendo a capacidade de alcançar o nível psicológico do sujeito acarretando em desmotivação, depressão e baixa autoestima. Por mais que o brasil seja um país miscigenado, a discriminação racial está muito presente no dia a dia dos brasileiros, causando intenso impacto na saúde mental das pessoas que sofrem preconceito, o que influência negativamente a autoestima e a auto-rejeição das vítimas. Pode-se perceber assim o impacto psicológico sofrido e esse sofrimento quando exacerbado pode levar os indivíduos ao ato suicida (SILVA, 2017).

\section{CONCLUSÃO}

É notável que o Brasil já evoluiu bastante frente às questões raciais nos últimos anos, porém algumas questões ainda estão estruturadas em nossa sociedade atual, como o racismo institucional presente nas diferentes esferas 
sociais. Assim, é frequente que muitas pessoas negras enfrentam dificuldades no acesso ao sistema de saúde, as quais são decorrentes de diversos fatores, como as discriminações nos serviços por parte de alguns profissionais, o preconceito dirigido aos próprios profissionais negros, ao mito de que as pessoas negras são mais resistentes as doenças e a dor, além das dificuldades econômicas, como moradia e alimentação.

Por mais que o Brasil seja um país miscigenado, infelizmente ainda se faz presente a existência de um racismo enraizado em nossa sociedade, em pleno século XXI, acarretando em profundos impactos psicológicos nessa população. Diante disso, se faz importante uma atenção voltada à saúde mental desses indivíduos, visando um apoio psicológico que auxilie em um tratamento desses possíveis impactos. Além disso, como já foi abordado, é importante que haja uma identificação entre paciente e profissional no atendimento terapêutico, possibilitando, dessa forma, que a pessoa atendida sinta-se mais confortável para abordar assuntos referentes às questões raciais.

Pode-se concluir que embora existam diversas políticas públicas de saúde voltadas para a população negra no Brasil, com a intenção de garantir o princípio da equidade assegurado pelo SUS, ainda percebe-se inúmeros desafios enfrentados por este grupo no acesso ao sistema de saúde. Neste sentido, vale destacar que o país ainda carece de um monitoramento contínuo sobre essas políticas para saber como elas estão sendo empregadas na prática. Assim, se faz necessária a implementação de uma fiscalização eficaz, para que o direito da população negra de ter uma saúde de qualidade seja garantido.

\section{REFERÊNCIAS}

BARDIN, L. Análise de conteúdo. São Paulo: Edições 70, 2016. Disponível em: https://madmunifacs.files.wordpress.com/2016/08/anc3a1lise-de-contec3bado-lauren ce-bardin.pdf. Acesso em: 02 abr. 2021. 
BRASIL. Ministério da Saúde. Portaria n 992, de 13 de maio de 2009. Institui a

Política Nacional de Saúde Integral da População Negra. Disponível em: https://bvsms.saude.gov.br/bvs/saudelegis/gm/2009/prt0992_13_05_2009.html. Acesso em: 27 ago. 2021.

BRASIL. Ministério da Saúde. Secretaria de Gestão Estratégica e Participativa. Departamento de Apoio à Gestão Participativa e ao Controle Social. Política Nacional de Saúde Integral da População Negra: uma política para o SUS. Ministério da Saúde, Secretaria de Gestão Estratégica e Participativa, Departamento de Apoio à Gestão Participativa e ao Controle Social. 3. ed. Brasília: Editora do Ministério da $2017 . \quad$ Saúde, Disponível em: https://bvsms.saude.gov.br/bvs/publicacoes/politica_nacional_saude_populacao_neg ra_3d.pdf. Acesso em: 30 mar. 2021.

BRASIL. Ministério da Saúde. Óbitos por suicídio entre adolescentes e jovens negros 2012 a 2016. Universidade de Brasília, 2018. Disponível em: http://bvsms.saude.gov.br/bvs/publicacoes/obitos_suicidio_adolescentes_negros_20 12_2016.pdf?fbclid=IwAR1JvKQluNZNIT6s_XKYEm6OiAUWfWH1toENITr1xUB1TjV _wIWCeA1iBIM. Acesso em: 30 mar. 2021.

COSTA, S.J. Saúde da população negra: Reflexões da Política Nacional de Saúde Integral da População Negra. Monografia (Graduação). Universidade Federal de Ouro Preto. Instituto de Ciências Sociais Aplicadas. Departamento de Ciências Sociais, Jornalismo e Serviço Social. Ouro Preto, dez, 2019. Disponível em: https://www.monografias.ufop.br/bitstream/35400000/2490/11/MONOGRAFIA_Sa\%c 3\%badePopula\%c3\%a7\%c3\%a3oNegra.pdf. Acesso em: 03 abr. 2021.

FAUSTINO, D. M. A universalização dos direitos e a promoção da equidade: o caso da saúde da população negra. Ciência \& Saúde Coletiva, v. 22, n. 12, 2017. Disponível em: https://www.scielo.br/pdf/csc/v22n12/1413-8123-csc-22-12-3831.pdf. Acesso em: 31 mar. 2021. 
GONÇALVES, W; COELHO, D.C; VIEIRA, A.S; SILVA, P.C.D; FILIIPE, A.R.T.M; SHITUKA, R. Danos causados pelo racismo por meio de termos linguísticos na saúde mental da população negra e a importância da psicologia preta para esse público: uma educação para as escolas. Revista de Casos e Consultoria, v.11, n. 1 , 2020.

Disponível

em: https://periodicos.ufrn.br/casoseconsultoria/article/view/22407/13452. Acesso em: 05 abr. 2021.

GUIMARAES, A. S. A. O insulto racial: as ofensas verbais registradas em queixas de discriminação. Estud. afro-asiáticos. Rio de Janeiro, n. 38, p. 31-48, Dec. 2000. Disponível em: <http://www.scielo.br/scielo.php?script=sci_arttext\&pid=S0101-546X2000000200002 \&lng=en\&nrm=iso>. Acesso em: 04 abr. 2021.

IBGE. Pesquisa nacional de saúde: 2013: Acesso e utilização dos serviços de saúde, acidentes e violências: Brasil, grandes regiões e unidades da federação. Coordenação de Trabalho e Rendimento. Rio de Janeiro: IBGE, 2015. Disponível em: ftp://ftp.ibge.gov.br/PNS/2013/pns2013.pdf. Acesso em: 30 mar. 2021.

KALCKMANN, S; SANTOS, C. G; BATISTA, L. E; CRUZ, V. M. Racismo institucional: um desafio para a eqüidade no SUS?. Saúde social, São Paulo , v. 16, n. 2, p. 146-155, ago. 2007 . Disponível em: <http://www.scielo.br/scielo.php?script=sci_arttext\&pid=S0104-12902007000200014 \&lng=en\&nrm=iso>. Acesso em 04 abr. 2021

MESQUITA, I.N. Entre silenciamentos e provocações: a questão do racismo no contexto da atenção básica à saúde. Unicamp: FCM: Campinas, 2019. Disponível em:

https://www.fcm.unicamp.br/fcm/sites/default/files/2019/page/tcc_igor_do_nasciment o_mesquita.pdf. Acesso em: 19 abr. 2021. 


\section{QUFN}

LAGES, S. R. C; TAVARES, N. O; SANTOS, S. V; CARVALHO, M. A. S; MACIEL, L. F. R. Pesquisas em Psicologia Social no Campo da Saúde da População Negra no Brasil. Revista de Psicologia da UNESP, 13(1). 2014 Universidade Federal de Minas Gerais. Disponível em: http://pepsic.bvsalud.org/pdf/revpsico/v13n1/a01.pdf. Acesso em: 31 de mar. 2021.

SANTOS, V. C;MORAIS, A. C; CARVALHO, E. S. S; SANTOS, J. S;SILVA,I.A.R; TEIXEIRA, J. B. C. Saúde da população negra no contexto da pandemia da covid-19: uma revisão narrativa. Brazilian Journal of Development, Curitiba, v.7, n.1, p. 2306-2320 jan. $2021 . \quad$ Disponível em: https://www.brazilianjournals.com/index.php/BRJD/article/view/22800 Acesso em: 13 maio 2021.

SILVA, M. C. O Impacto do Racismo na Saúde Mental das Vítimas. Psicologia.pt : o portal dos psicólogos, 2017. Disponível em: https://www.psicologia.pt/artigos/textos/A1229.pdf. Acesso em: 04 abr. 2021.

SILVA, N. N.; Favacho, V. B. C.; BOSKA, G. A.; ANDRADE, E. C.; MERCES, N. P.; OLIVEIRA, M. A. F. Acesso da população negra a serviços de saúde: revisão integrativa. Revista Brasileira de Enfermagem. v. 73, n. 4, p. e20180834. 2020 DOI: http://dx.doi.org/10.1590/0034-7167-2018-0834. Disponível: https://www.scielo.br/scielo.php?script=sci_arttext\&pid=S0034-71672020000400301 \&lng=en\&nrm=iso\&tlng=pt. Acesso em: 01 abr. 2021. 